Versuche mit Aceton.

IV. Unter denselben Bedinguagen wie bei Versuch I und II wurden $2 \mathrm{~g}$ palladiniertes Nickelpulver (mit $0.09 \mathrm{~g}$ Pallacliom) mit $2.5 \mathrm{~g}$ des Esters, der in der fünffachen Menge A ceton gelöst war, zusammengebracht. In der ersten Stunde verschwanden $6 \mathrm{ccm}$ Wasserstoff, dann blieb das Gasvolumen konstant.

v. Eine Wiederholung des Versuchs unter den gleichen Bedingungen ergab dasselbe Resultat.

Während demnach Benzol und Aceton auf das palladinierte Nickel antikatalytisch wirken, ergaben unter den gleichen Bedindungen angestellte. Versuche, bei denen als Lösungsmittel Äther und Alkohol dienten, daß diese keine passivierende Wirkung auszuüben vermögen. Die Übertragung des Wasserstoffs auf den hierbei zu reduzierenden Ester durch das Palladium verlief glatt bis zu Ende.

Das verschiedene Verbalten von Benzol und Aceton einerseits, von Äther und Alkobol andererseits läßt sich durch die Annabme erklären, daß die Tendenz des Palladiums zur Adsorption der erstgenanuten Lösungsmittel größer ist als für Wasserstoff, während der vom Katalysator adsorbierte Äther oder Alkohol durch Wasserstoff verdrängt werden kann.

Die Versuche werden fortgesetzt.

136. Dan Radulescu: Synthese apyrocyclischer Verbindungen.

(Eingegangen am 22. März 1911.)

In einer vorläufigen Mitteilung ${ }^{1}$ ) habe ich eine Methode zur Synthese homocyclischer Spirane mit zwei Ketongruppen beschrieben. Diese Methode bestand darin, $\alpha, \alpha$-Polymethylen-dicarbonsäureester mit Bernsteinsäureester mittels Natriumamid zu kondensieren, um eine Substanz vom Schema I zu erhalten. An Stelle derselben erhielt ich ein saures, stickstoffhaltiges Produkt, das sich aus dem erwarteten Ester durch Verseifung und durch die Einwirkung des bei der Kondensation entstandenen Ammoniaks gebildet hatte.

Erst kürzlich konnte ich die experimentellen Bedingungen auffinden, unter deven man stickstofffreie Kondensationsprodukte erbalten kann. Gleichzeitig gelang es, die Ausbeute $\left(1 \frac{1}{2}-2 \%\right.$ ) zu verbessern und das Reaktionsprodukt in Form 2 weier Säuren (II), (III) zu isolieren.

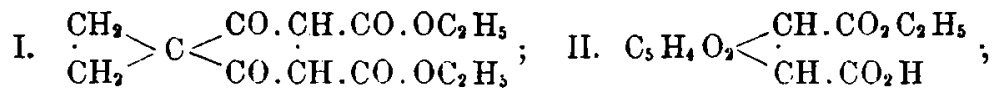
III. $\mathrm{C}_{5} \mathrm{H}_{4} \mathrm{O}_{2}<\stackrel{\mathrm{CH} \cdot \mathrm{COOH}}{\mathrm{CH} . \mathrm{COOH}}$.

1) B. 42, 2771 [1909]. 
Im Reaktionsprodukt sind die Natriumsalze der beiden Säuren und die Natriumverbindung des Succinylo-bernsteinsäureesters entbalten, dessen Bildung durch die Anwesenheit einer kleinen Menge Natriumalkoholat erklärt ist, das bei der gleichzeitig stattfindenden Verseifung entsteht. Das Gemenge der Natriumsalze wird in Waseer gelöst und läßt sich durch fraktionierte Fällung durch Säuren leicht in die einzelnen Komponenten zerlegen. Durch einmaliges oder höchstens zweimaliges Umkrystallisieren lassen sich für die Analyse genügend reine Produkte erhalten.

Die angegebene Konstitution ergibt sich deutlich aus der Synthese, der Zusammensetzung und den Eigenschaiten der Produkte.

Das Produkt (II) ist eine einbasische, das Produkt (III) eine zweibasische Säure. Die Estersäure (II) .ve̛rliert beim mohrmaligen Umkrystallisieren aus kochendem Wasser Alkobol und geht in die zweibasische Säure (III) über; diese ihrerseits verwandelt sich durch partielle Veresterung in die Estersäure (II).

Mit Eisenchlorid geben beide Säuren eine sebr empfindliche und intensive Violettfärbung, welche für $\beta$-Ketonsäuren charakteristisch ist.

Die Estersäure verliert beim Erhitzen suf $180-190^{\circ}$ Kohlensäure und verwandelt sich in ein schon früber ${ }^{1}$ ) beschriebenes blaues Produkt, dessen Konstitution noch völlig unbekannt ist. Wenn die zweibasische Säure auf dieselbe Temperatur erhitzt wird, erleidet sie unter Verlust von Kohlensäure teilweise dieselbe Umwandlung; der größere Teil geht aber unter Wasserabspaltung in ein weißes, unschmelzbares Anhydrid über, das in Alkohol und anderen organischen Lösungsmitteln unlöslich ist.

Beim Behandeln mit Alkali zersetzen sich beide Säuren fast quantitativ in Bernsteinsäure und Cyclopropan- $\alpha, \alpha$-dicarbonsäure in äquimolekularen Mengen. Sie addieren keine Hadogenwasserstoffsäuren. Durch Brom werden sie unter Entweichen von Strömen Bromwasserstoff zersetzt. Sie reagieren weder mit Phenylhydrazin, noch mit $\mathbf{H y}$ droxylamin.

Durch längeres Kochen mit verdünnter Schwefelsäure werden die Säuren in Bernsteinsäure und Cyclopropan- $\alpha, \alpha$-dicarbonsäure und ein flüchtiges Keton zersetzt, dessen Studium aus Mangel an Material noch nicht abgeschlossen ist.

Experimenteler Teil

Kondensation.

$\mathrm{Zu}$ einer Mischung von $37 \mathrm{~g}$ Cyclopropan- $\alpha, \alpha$-dicarbonsäureester und $35 \mathrm{~g}$ Bernsteinsäureester im fünffachen Volumen absoluten Äthers werden in einem mit Rückflußkühler versehenen Kolben, der von 
außen durch ein Wasserbad von $10-15^{\circ}$ gekühlt ist, 20 g Natriumamid als feines Pulver hinzugefügt. Die Mischung erwärmt sich; es entwejcht Ammoniak, und das Natriumamid verschwindet allmäblich unter Verwandlung in ein kreidiges Produkt, das sich absetzt. Nach 24 Stunden hört die Entwicklung von Ammoniak auf, und die Reaktion ist beendigt. Nach dem Zufügen einer Mischung ron $3 \mathrm{Tln}$. zerstossenem Eis und 2 Tln. Wasser wird geschüttelt, bis alles gelöst ist. Dann wird die wäßrige, gelbe Lösung ron der ätherischen, schwach blau fluorescierenden getrennt und nach dem Abfiltrieren von einigen organischen Verunreinigungen mit 1/8 Mol. konzentrierter Salzsäure und $3 \mathrm{Tln}$. zerstoßenem Eis behandelt. Man schüttelt gut und trennt dann so schnell wie möglich durch Absaugen auf einem großen Filter ron dem gelblichen Niederschlag, der aússchließlich aus Succinylo-bernsteinsäureester bestebt. Dann fiigt man, obne zu warten, zu dem Filtrat, das sich von neuem zu trüben beginnt, noch ${ }_{1 / 8}$ Mol. Salzsäure wie vorber hinzu. Nach einer Viertelstunde filtriert man die abgeschiedene Estersäure ab und versetzt noch mit 1/4 Mol. Salzsäure. Es scheidet sich dann in 48 Stunden der größte Teil der zweibasischen Säure aus. Durch Konzentrieren unter vermindertem Druck kann man noch eine kleine Menge isolieren.

Aus der ätherischen Schicht kann man neben unangegriffenen Estern kleine Menge Succinylo-bernsteinsäureester und manchmal etwasBernsteinsäureimid isolieren.

Monäthylester des Cyclopropan-cyclopentan-2.5-dion-3.4dicarbonsäure-(1.1)-spirans.

Der weiße, krystallinische Niederschlag, der bei der zweiten Fraktion erhalten wurde, stelit nach ein- oder böchstens 2 weimaligem Umkrystallieren aus wenig kochendem ${ }^{2}$ ) Wasser feine, durchscheinende, seidenglänzende Schuppen dar, welche bei $153-154^{\circ}$ unter Zersetzung (Entwicklung von Kohlensäure) schmelzen und dann wieder erstarren, ohne sich ein zweites Mal zu verflüssigen. Bei $180-190^{\circ}$ färben sio sich blau und zersetzen sich vollständig bei $250^{\circ}$. Ausbeute $0.8-1.5 \mathrm{~g}$.

$0.1392 \mathrm{~g}$ Sbst.: $0.2810 \mathrm{~g} \mathrm{CO}_{2}, 0.0647 \mathrm{~g} \mathrm{H}_{2} \mathrm{O} .-0.1410 \mathrm{~g}$ Sbst.: $0.2849 \mathrm{~g}$ $\mathrm{CO}_{2}, 0.0638 \mathrm{~g} \mathrm{H}_{2} \mathrm{O}$.

$$
\begin{aligned}
& \mathrm{C}_{11} \mathrm{H}_{12} \mathrm{O}_{6} \text {. Ber. C 55.00, H 5.00. } \\
& \text { Gel. } \$ 55.05,55.11 \text {, 5.21, 5.07. }
\end{aligned}
$$

1) Man darf die Lösung nicht lange im Sieden erhalten, muß schnell abkühlen und darf für eine Operation nicht mehr als $1.5 \mathrm{~g}$ der Săure in 25$30 \mathrm{ccm}$ Wasser verwenden. Häufige Krystallișationen and unvorsichtige Operationen verunreinigen nur das Produkt. 
Die Substanz ist leicht löslich in Alkohol, Aceton, Methylalkobol, weniger löslich in Äther, Chloroform, Benzol, unlöslich in Petroläther. In heißem Wasser löst sie sich leicht und ist beim Abkühlen krystallinisch 20 gewinnen.

Mit Phenolphthalein als Indicator verhält sich die Substanz als einbasische Säure. $0.500 \mathrm{~g}$ Sbst. brauchen zur Neutralisation $21.1 \mathrm{ccm}$ $0.1-n$. Kalilauge, ber. $20.8 \mathrm{ccm}$.

Die neutrale Lösung des Kaliumsalzes gibt mit Bariumchlorid eine Abscbeidung, mit Bleinitrat einen weißen, käsigen Niederschlag; mit Silbernitrat ist er weiß und zersetzlich, mit Kupfersulfat hellgrăn und mit Eisenchlorid violettschwarz.

\section{Quantitative Zersetzung.}

$0.24 \mathrm{~g}$ Sbst. wurden in $3-4 \mathrm{ccm}$ 60-prozentiger chlorfreier Kalilauge gelöst und ca. 2 Stunden auf dem Wasserbade erwärmt. Nach dem Abkühlen mit Eis wird mit verdünnter kalter Schwefelsäure angesäuert und 15-mal mit kleinen Portionen Äther :extrahiert. Der saure Rückstand aus dem Ätherextrakt wird 5 Minuten in einem Kolben mit langem $\mathrm{Hals}$ in Ölbad auf $170-180^{\circ}$ erhitzt, wobei Kohlensäure entweicht; dann wird er in $30 \mathrm{ccm} n$-Kalilauge gelöst, genau mit verdünnter Salpetersăure neutralisiert und die Lösung auf $60^{\circ}$ erwärmt. Darauf wird mit einem geringen Überschuß von Bariumnitrat gefällt, das bernsteinsaure Barium abfiltriert, wieder in Chlorwasserstoffsäure gelöst und nach dem Abfiltrieren geringer Mengen Bariumsulfat, die von kleinen Mengen Schwefelsäure herrübren, welche mechaniscb während der Extraktion aufgenommen waren, in der filtrierten Lösung das Barium als Bariumsulfat bestimmt. Man hat so die Bernsteinsäure ermittelt. Im Filtrat vom bernsteinsauren Barium fält man die Cyclopropan-monocarbonsäure als Silbersalz, wäscht und glibt es und hat auf diese Weise die Säure bestimmt.

$0.24 \mathrm{~g}$ Sbst.: $0.2275 \mathrm{~g} \mathrm{BaSO}_{4}, 0.0983 \mathrm{~g} \mathrm{Ag}$.

Ber. für $0.001 \mathrm{Mol}$. $\mathrm{C}_{4} \mathrm{H}_{6} \mathrm{O}_{4}: 0.1180 \mathrm{~g}, \mathrm{C}_{4} \mathrm{H}_{6} \mathrm{O}_{2}: 0.0860 \mathrm{~g}$

Gef. " > > $0.1150 \mathrm{~g}$, > $0.0782 \mathrm{~g}$.

Wenn man die wäßrige Lōsung des Produkts einige Zeit lang zum Sieden erhitzt, färbt sie sich rot mit grüner Fluorescenz, und beim Abkühlen krystallisiert in schönen, farblosen Nadeln ein Körper aus, der bei $161^{\circ}$ schmilzt und nichts anderes ist als das

\section{Cyclopropan-cyclopentan-2.5-dion-3.4-dicarbonsäure- (1.1)-spiran.}

Die dritte, viel reichlichere Fällung als die beiden anderen, besteht ausschlieBlich aus dieser Säure. Beim zweimaligen Umkrystal- 
lisieren aus siedendem Wasser erbält man sie in Form einer Krystallmasse von blattförmigen Nadeln. Nach dem Trocknen über Schwefelsäure enthält sie noch ein Molekül Krystallwasser, und schmilzt bei $161^{\circ}$ unter teilweiser Zersetzung, wobei sie sich unter Verlust von Wasser aufbläht. Bei einer etwas höheren Temperatur verwandelt sie sich in eine Mischung des blauen Produktes (siehe oben) und eines körnigen Anhydrids, das in allen organischen Lösungsmitteln unlöslich ist und nicht ohne Zersetzung schmilzt.

Beim Erhitzen im Vakuum auf $70-80^{\circ}$ verliert die Säure über Phosphorpentoxyd allmählich ibr Krystallwasser, es findet aber gleichzeitig eine Zersetzung unter Verlust von Kohlensäure statt.

$0.2344 \mathrm{~g}$ Şbst. über $\mathrm{H}_{3} \mathrm{SO}_{4}$ getrocknet: $0.4024 \mathrm{~g} \mathrm{CO}_{2}, 0.0997 \mathrm{~g} \mathrm{H}_{2} \mathrm{O}$. $0.1998 \mathrm{~g}$ Sbst.: $0.3435 \mathrm{~g} \mathrm{CO}_{2}, 0.0787 \mathrm{~g} \mathrm{H}_{2} \mathrm{O}$.

$$
\begin{aligned}
& \mathrm{C}_{9} \mathrm{H}_{8} \mathrm{O}_{6}+\mathrm{H}_{2} \mathrm{O} . \text { Ber. C 46.96, } \quad \text { H } 4.35 . \\
& \text { Gef. > } 46.82,46.89, 》 4.76,4.42 .
\end{aligned}
$$

$0.23 \mathrm{~g}$ Sbst. ergaben bei der Behandlung mit Kali (wie oben): $0.2196 \mathrm{~g}$ $\mathrm{BaSO}_{4}$ und $0.0979 \mathrm{~g} \mathrm{Ag}$.

Ber. für $0.001 \mathrm{Mol} . \mathrm{C}_{4} \mathrm{H}_{6} \mathrm{O}_{4}: 0.1180 \mathrm{~g}, \mathrm{C}_{4} \mathrm{H}_{6} \mathrm{O}_{2}: 0.0860 \mathrm{~g}$.
Gef. $" 》 0.1110 \mathrm{~g}, \geqslant 0.0779 \mathrm{~g}$.

$0.23 \mathrm{~g}$ der' Sāure verlangen $20 \mathrm{ccm}$ 0.1-n. KOH zur Neutralisation, gef. $19.5 \mathrm{ccm}$.

Die neutrale Lösung des Kaliumsalzes gibt mit Bariumchlorid einen krystallinischen, mit Bleinitrat einen weißen, voluminösen, mit Eisencblorid einen violettschwarzen, mit Silbernitrat einen weißlichgrauen, später schwarz werdenden und mit Kupfersulfat einen grünen Niederschlag.

\section{Bildung des Esters.}

Ein Teil der Säure wurde in 5 Tln. absolutem Alkohol gelöst und mittels Chlorwasserstoff verestert. Man befreit das Reaktionsprodukt durch Verdünnen mit Äther und Schütteln mit Natriumbicarbonat von der Säure. Der Rückstand der ätheriscb-alkoholiscben Lösung stellt nach zweimaligem Umkrystallisieren aus Wasser die bei $152^{\circ}$ schmelzende Estersäure dar.

Chemisches Institut der Universität Berlin, 7. März 1911. 